\title{
A Differential Optical Sensor for Non-Invasive Real-Time Monitoring of Ultrafiltration Rate in Hemofiltration Therapies
}

\author{
Andrea Visotti ${ }^{\dagger}$, Enrico Ravagli ${ }^{\dagger}$, Claudia Perazzini, Debora Drudi, Corrado Ghidini and Stefano Severi*
}

\begin{abstract}
Hemofiltration (HF) is a group of blood purification therapies used to treat patients with kidney injury. HF works using a process called ultrafiltration (UF) that removes excess liquid accumulated in the patient's body caused by lack of excretion. UF progress is monitored by the HF machine, but the state-of-the-art method is cumbersome and could be more accurate. In this work, a system composed by two optical sensors is proposed for real-time non-invasive estimation of ultrafiltration rate. This new system is simple, rugged, low-cost and operates on sound theoretical foundations. The sensor system has been tested with two different experimental protocols and showed good correlation between its output and the reference value of the ultrafiltration rate $\left(R^{2}=0.97\right)$, as well as improved accuracy compared to the available commercial machine $(\simeq 12 \mathrm{ml} / \mathrm{h})$. This system also has the potential to simplify the architecture required by critical care blood purification machines to perform UF control.
\end{abstract}

Index Terms - Biomedical Measurement, Blood, Blood Purification, Hemofiltration, Hemoglobin, Measurement, Optical Sensor, Ultrafiltration

\section{INTRODUCTION}

Renal replacement therapies (RRTs) have been developed to treat individuals affected by kidney failure, known as nephropathic patients. RRTs have the following targets:

- Removal of excess fluid accumulated by the patient due to lack of kidney excretion.

- Re-balancing concentration of specific substances in blood, for example electrolytes such as sodium.

- Removal of the toxic by-products of metabolism, such as urea.

Several types of therapies and machines have been developed. The main distinction of therapies is between those developed

†First two authors A. Visotti and E. Ravagli contributed equally to this work.

A. Visotti, C. Perazzini, D. Drudi, and C. Ghidini are with the company IBD Srl (https://www.ibdsrl.com/), Forlì, Italy.

E. Ravagli and S. Severi* are with the Department of Electrical, Electronic and Information Engineering "Guglielmo Marconi”, University of Bologna, Cesena, Italy (e-mail: stefano.severi@unibo.it). to treat patients with acute kidney injury (AKI), who are expected to partially or fully regain kidney functionality, and those for patients with chronic kidney disease (CKD), who require periodic treatment for the rest of their lives or until kidney transplant. Therapies such as hemodialysis [1]-[3] and peritoneal dialysis [4] are the standard for CKD patients; while for AKI, slow, prolonged, one-time treatments are chosen to perform blood purification until kidney function is regained [5]-[7]. The effect of RRTs on the patient has been studied both clinically and with mathematical tools such as kinetic models, developed to describe the transfer of water and solutes across body compartments during RRTs [8]-[10].

This work represents a technological improvement for AKI therapies where fluid removal is the main target - therapies also known as hemofiltration (HF). Fluid removal is performed by means of a physical principle called ultrafiltration (UF) [11]-[13]. Examples of specific hemofiltration therapies are slow continuous ultrafiltration (SCUF), continuous artero-venous (CAVH) and veno-venous hemofiltration $(\mathrm{CVVH})$ [14]. Since fluid removal is the main target of these therapies, the process is monitored by technological means to ensure a stable ultrafiltration rate (UFR) and the achievement of the desired end-session target weight loss, usually by performing sensing and closed-loop control on UFR.

This paper describes the development of a new type of sensor for hemofiltration RRTs. The sensor takes advantage of a differential optical measurement principle to measure UFR in a non-invasive manner, so that the system does not come into direct contact with blood. The new sensor has the potential of improving the accuracy of UFR estimation, and consequently its control, as well as simplifying the machine architecture.

In section II, basic theoretical background on HF, UF and optical sensing is outlined. Section III describes the theoretical principles and technical aspects of the system, including a description of the experimental setup and protocols used for validation. Experimental results are reported in section IV. In section $\mathrm{V}$, the results are analyzed, and current limitations of the system and probable future developments are discussed. 


\section{THEORETICAL BACKGROUND}

\section{A. Hemofiltration and Ultrafiltration}

RRT therapies work by withdrawing blood from the patient, purifying it during extracorporeal circulation (EC), then reinjecting it. Purification is performed by means of a filter, the dialyzer, placed along the EC circuit. In the case of hemofiltration, only fluid removal is performed at the filter, whereas re-injection of sterile fluid with different solutes is eventually performed further downstream. When the patient only needs treatment for fluid overload, no fluid re-injection is performed, corresponding to an isolated UF treatment [13].

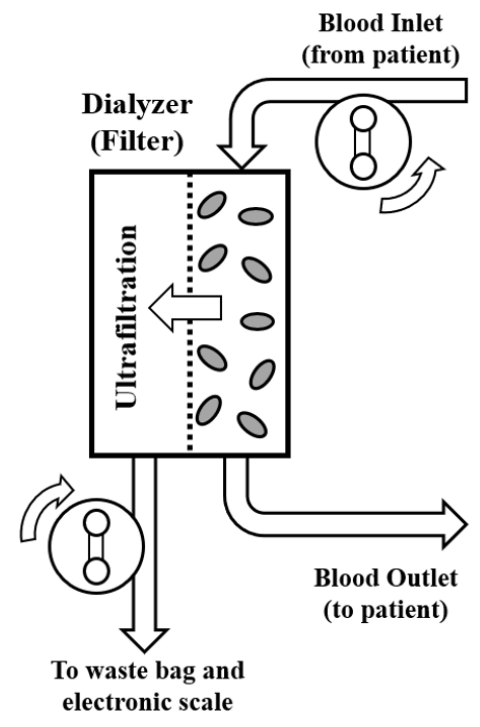

Fig. 1. Simplified diagram of the hemofiltration process.

Fig. 1 shows a simplified diagram of the hemofiltration process. Blood withdrawn from the patient flows along the extracorporeal path across the dialyzer by means of two roller pumps, while a third pump on the other side of the filter applies a negative pressure gradient across the dialyzer membrane, withdrawing water and small solutes but no large molecules or red blood cells. This process is called ultrafiltration. Waste liquid is collected in a bag, which is removed by the operator after the session. The diagram in Fig. 1 is conceptual; a real HF set-up would also include additional pumps along the circuit for a range of purposes such as the injection of the anticoagulant (e.g. heparin) or replacement fluid (pre/post-dilution pumps).

The ultrafiltration rate (UFR) is typically set either through the roller pump encoder and rounds-per-minute (rpm) settings, or by monitoring differential pressure across the hemodialyzer's membrane and regulating the pump accordingly. The specific implementation is machinedependent. A secondary check is performed by an integrated electronic scale, which weighs the collected waste liquid in order to assess treatment progression, detect possible issues and correct UFR if needed.

However, this method has drawbacks. The electronic scale is very sensitive to perturbations to the hanging waste bag, which may affect readings; the operator must take extreme care not to touch the bag during the session. Also, the waste bag must be replaced multiple times during the session, and during these times the UF process must be temporarily stopped. Thus, the present system for UFR control is encumbering and stressful for the operator. Accuracy for this kind of method of UFR control is approximately in the tens of $\mathrm{ml} / \mathrm{h}$; for example, the machine used in this research has a documented accuracy of $30 \mathrm{ml} / \mathrm{h} \mathrm{[15].}$

\section{B. Optical sensors in hemodialysis and hemofiltration}

This work reports a novel system for HF treatments based on the application of sensors typically used on HD machines for different purposes. In this subsection, a brief description of such sensors is given.

Several sensors are embedded on HD machines for on-line monitoring of relevant parameters and for patient safety, with new designs being periodically reported in literature [16][19]. Non-invasive sensors are particularly useful to avoid the expense of disposable material and probes [16], [18], [20], [21]. Optical sensors are mainly used for the purpose of relative blood volume (RBV) estimation [22], [23], but other uses have also been proposed [16], [17], [19], [24], [25]. Relative blood volume is defined as the relative variation in volume of the body's blood compartment in comparison with that at the beginning of the session. It is determined by the time-dependent balance between the UFR and the refilling rate (RR), which is the rate at which the patient physiologically refills the circulatory system with liquid coming from the extravascular space [26] to avoid a drop in blood pressure. Equation (1) shows the mathematical formulation for RBV, where $t_{0}$ is the time at which the HD session starts.

$$
R B V(t)=\frac{V_{B}(t)}{V_{B}\left(t_{0}\right)}=\frac{V_{B}\left(t_{0}\right)-\int_{t 0}^{t} U F R(t)-R R(t)}{V_{B}\left(t_{0}\right)}
$$

Knowledge of RBV is useful for the purpose of patient-based therapy customization: in the past, biofeedback algorithms were developed to tune HD session parameters in real-time on the basis of physiological variables [27]-[31]. For example, UFR can be modulated in real-time on the basis of an RBV estimate to avoid hypotensive events [29]. However, the blood volume at the session start $\mathrm{V}_{\mathrm{B}}\left(\mathrm{t}_{0}\right)$ is not known and its measurement would be impractical and invasive. Thus, RBV is calculated starting from the blood hemoglobin concentration, as shown in Eqs. (2-3). In Eq. (3) it is assumed that hemoglobin mass does not change significantly during the session, only blood volume does.

$$
\begin{aligned}
& {[H g b](t)=\frac{m_{H g b}}{V_{B}(t)}} \\
& R B V(t)=\frac{[H g b]\left(t_{0}\right)}{[H g b](t)}=\frac{m_{H g b}}{V_{B}\left(t_{0}\right)} \cdot \frac{V_{B}(t)}{m_{H g b}}=\frac{V_{B}(t)}{V_{B}\left(t_{0}\right)}
\end{aligned}
$$

In this context, optical sensing is used for the estimation of hemoglobin concentration in blood. The typical architecture of an optical sensor for HD is shown in Fig. 2.

In this setup, a light source and a detector are placed across 


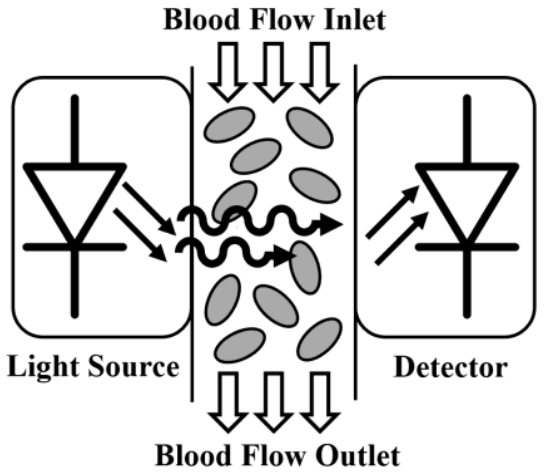

Fig. 2. Simplified diagram of a typical optical blood volume sensor for hemodialysis.

the blood flow circuit - the bloodline - and light is transmitted across the bloodstream, usually across a special optically transparent section of the bloodline - the cuvette. The light source is typically a light-emitting diode (LED) and the detector is usually a photodiode. Light is emitted with a central wavelength of $\simeq 810 \mathrm{~nm}$. At this wavelength, hemoglobin has the same optical absorbance regardless of its oxygen content. The relationship between transmitted light power $\mathrm{P}_{\mathrm{TX}}$, received power $\mathrm{P}_{\mathrm{RX}}$ and hemoglobin concentration $[\mathrm{Hgb}]$ is regulated by Lambert-Beer's equation, reported in eq. (4).

$P_{R X}=P_{T X} \cdot e^{-\alpha \cdot[H g b] \cdot d}$

In the above equation, $\alpha$ is the molar attenuation coefficient and $d$ is the optical path length. Thus, by knowing $\mathrm{P}_{\mathrm{TX}}, d$ and $\alpha,[\mathrm{Hgb}]$ can be measured by optical means and used in the determination of $\mathrm{RBV}(\mathrm{t})$ in real-time according to Eq. (3). The real-world implementation of this type of sensor also includes calibration coefficients for practical issues such as voltage offsets and gain error in the electronics.

\section{MATERIALS AND METHODS}

\section{A. Measurement Principle}

The optical measurement described in Section II.B is usually performed on HD machines for treatment of CKD patients. In the new system, this type of measurement is performed on machines for HF treatments in two distinct positions along the EC blood path: before and after the hemodialyzer. The difference between the two sensor outputs is quantitatively related to the physical effect that occurs in the dialyzer, i.e. ultrafiltration. Thus, the setup acts as a noninvasive ultrafiltration rate sensor.

The outputs of the two sensors are formalized in Eqs. (5-6), where $\mathrm{K}$ is a positive coefficient taking into account both the geometrical coupling between light source and detector and the transimpedance gain of the photodiode reading circuitry. It is assumed that coefficient $\mathrm{K}$, emitter intensity $\mathrm{P}_{\mathrm{TX}}$, and optical path length $d$ are all equal for the two sensors: slight differences due to fabrication, tolerance of components and setup preparation are considered negligible. Molar attenuation coefficient $\alpha$ is by definition also the same.
$V_{1}=K \cdot P_{R X, 1}=K \cdot P_{T X} \cdot e^{-\alpha \cdot[\mathrm{Hgb}]_{1} \cdot d}$

$V_{2}=K \cdot P_{R X, 2}=K \cdot P_{T X} \cdot e^{-\alpha \cdot[H g b]_{2} \cdot d}$

However, concentrations $[\mathrm{Hgb}]_{1}$ and $[\mathrm{Hgb}]_{2}$ will be different: the hemoglobin mass, $\mathrm{m}_{\mathrm{Hgb}}$ in Eq. (2), will be distributed in a smaller volume post-filter due to the removal of fluid across the filter's membrane. Thus, $[\mathrm{Hgb}]_{2}$ will be slightly higher than $[\mathrm{Hgb}]_{1}$, and transmitted light will be decreased, reducing $\mathrm{V}_{2}$ compared to $\mathrm{V}_{1}$. Assuming [Hgb] variations during the sessions are small, equation for $\mathrm{V}_{2}$ can be linearized around $[\mathrm{Hgb}]_{1}$, leading to:

$$
\begin{aligned}
& V_{2}=V_{1} \cdot e^{-\alpha \cdot\left([H g b]_{2}-[H g b]_{1}\right) \cdot d} \simeq \\
& \simeq V_{1} \cdot\left(1-\alpha \cdot d \cdot\left([H g b]_{2}-[H g b]_{1}\right)\right)
\end{aligned}
$$

The assumption of small [Hgb] variations is acceptable as long as blood volume loss is limited, as expected during the session. The magnitude of blood volume loss that would invalidate this assumption would be associated with additional complications (i.e. hypotension, fainting), leading to the interruption of the session and thus making the sensor output irrelevant.

The difference between signals $\mathrm{V}_{1}$ and $\mathrm{V}_{2}$ then assumes the following form:

$V_{\text {Diff }}=V_{1}-V_{2} \simeq V_{1} \cdot(\alpha \cdot d) \cdot\left([H g b]_{2}-[H g b]_{1}\right)$

Given that $m_{\mathrm{Hgb}}$ is assumed constant, $[\mathrm{Hgb}]_{1}-[\mathrm{Hgb}]_{2}$ will only be influenced by the effect of UFR on blood volume $V_{B}$. In this report, we assume for simplicity that:

$\left([\mathrm{Hgb}]_{2}-[\mathrm{Hgb}]_{1}\right)=c \cdot U F R$

where c is a generic positive constant. Equation (9) is physically sound, as an increase in ultrafiltration rate will also increase the disparity of hemoglobin concentration across the filter by increasing $\left[\mathrm{Hgb}_{2}\right.$. Eqs. (8) and (9) can be combined and normalized in respect to $\mathrm{V}_{1}$, resulting in an index linearly related to UFR:

$I_{U F R}=\frac{V_{D i f f}}{V_{1}}=\frac{V_{1}-V_{2}}{V_{1}} \simeq(\alpha \cdot d \cdot c) \cdot U F R=K^{\prime} \cdot U F R$

In Eq. (10) above, $\mathrm{K}^{\prime}$ is a positive coefficient used to aggregate all constant parameters. It should be highlighted that measurement of $V_{1}$ and $V_{2}$ or that of $V_{1}$ and $V_{\text {Diff }}$ are both theoretically equivalent for the computation of the equation, however, $\mathrm{V}_{\text {Diff }}$ is a very small signal compared to $\mathrm{V}_{1}$ and $\mathrm{V}_{2}$, and its time-dependent evolution may not be detectable if this signal is computed as the difference of previously digitized $V_{1}$ and $\mathrm{V}_{2}$. Thus, in our measurement setup $\mathrm{V}_{\text {Diff }}$ is sampled as an analogue voltage signal in a differential configuration between the $V_{1}$ and $V_{2}$ voltages, while $V_{1}$ is sampled independently as a single-ended signal. Afterwards, I IfR can be calculated and the UFR determined from an empirically estimated value of K'. 


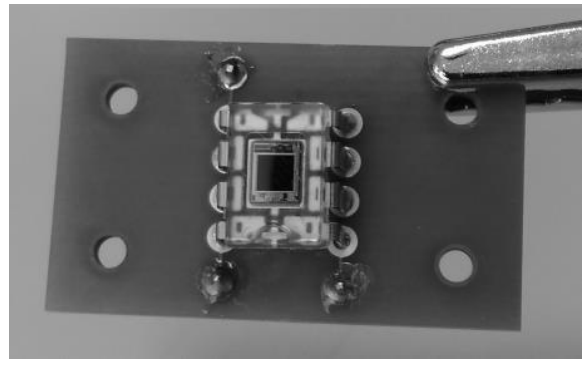

Fig. 3. Optical sensor module.

\section{B. Measurement Setup}

Measurement setup is composed of four parts:

- Two sensor modules placed on the bloodline, before and after the dialyzer

- A through-hole circuit board

- A microcontroller-based prototyping board

Each of the sensor modules is composed of a light-emitting diode (LED) light source and a photodiode with an integrated transimpedance amplifier. The LED and the photodiode are placed on opposite sides of a custom 3D-printed plastic housing, allowing the fitting of an optical flow-through cuvette between the two components to measure transmitted light. The photodiode is soldered on a small printed circuit board (PCB) to allow proper placement of the component and provide easier access to its pins. LEDs with a central emission wavelength of $810 \mathrm{~nm}$ are used (MTE1081C, Marktech Optoelectronics, Latham, New York, USA). An OPT101 photodiode and transimpedance amplifier (Texas Instruments, Dallas, Texas, USA) is used to convert light transmitted across the bloodline directly to a voltage output signal. The photodiode is set to its default configuration to allow for maximum gain. One of the sensor modules is shown in Fig. 3.

Given our measurement principle, the physical location of the sensor modules is extremely relevant. As explained in the previous subsection, sensor modules are placed immediately before and after the hemodialyzer on the EC circuit. This way, the only physical quantity able to modify the properties of blood is UFR.
Sensor modules are connected to a circuit board designed to include a current regulator for the LEDs, low-pass filters for the voltage outputs of the photodiodes and an analogue-todigital converter (ADC) for the digitization of the signals. A CAT4104 constant current LED driver (ON Semiconductor, Phoenix, Arizona, USA) is used together with an external $6.8 \mathrm{~K} \Omega$ resistor to drive the LEDs with a regulated $18 \mathrm{~mA}$ current. Voltage outputs from the OPT101 photodiodes were processed by simple first-order low-pass analogue filters with a $5.3 \mathrm{~Hz}$ cut-off frequency prior to analogue-to-digital conversion. An ADS1115 (Texas Instruments, Dallas, Texas, USA) ADC was used to digitize the signals. This ADC was specifically chosen for its 16-bit resolution and its capability of switching between single-ended measurements and differential measurements on its physical channel using only software instructions. This feature was necessary in order to separately convert all the relevant analogue measurements $\left(\mathrm{V}_{1}, \mathrm{~V}_{2}\right.$ and $\left.\mathrm{V}_{\text {diff }}\right)$.

An Arduino UNO microcontroller-based prototyping board (https://www.arduino.cc) was used for the purpose of data collection and transmission and to supply the PCB with $5 \mathrm{~V}$ voltage. The internal ADC of the Arduino board was not used since it only has 12-bit resolution. The Arduino board was programmed to take consecutive measurements of each single sensor output, ground-referenced, as well as the differential voltage between the two voltage outputs. On the basis of preliminary measurements, the ADC voltage range was dynamically set to $\pm 4.096 \mathrm{~V}$ for the single-ended measurements and to $\pm 0.512 \mathrm{~V}$ for the differential measurement. The sampling frequency for each of the three signals was $16 \mathrm{~Hz}$. Collected data was transmitted from the Arduino board to a PC using the serial protocol over USB cable (virtual COM port). A custom interface developed in LabView (National Instruments, Austin, Texas, USA) was used for data visualization and storage.

A diagram of the complete system is shown in Fig. 4.

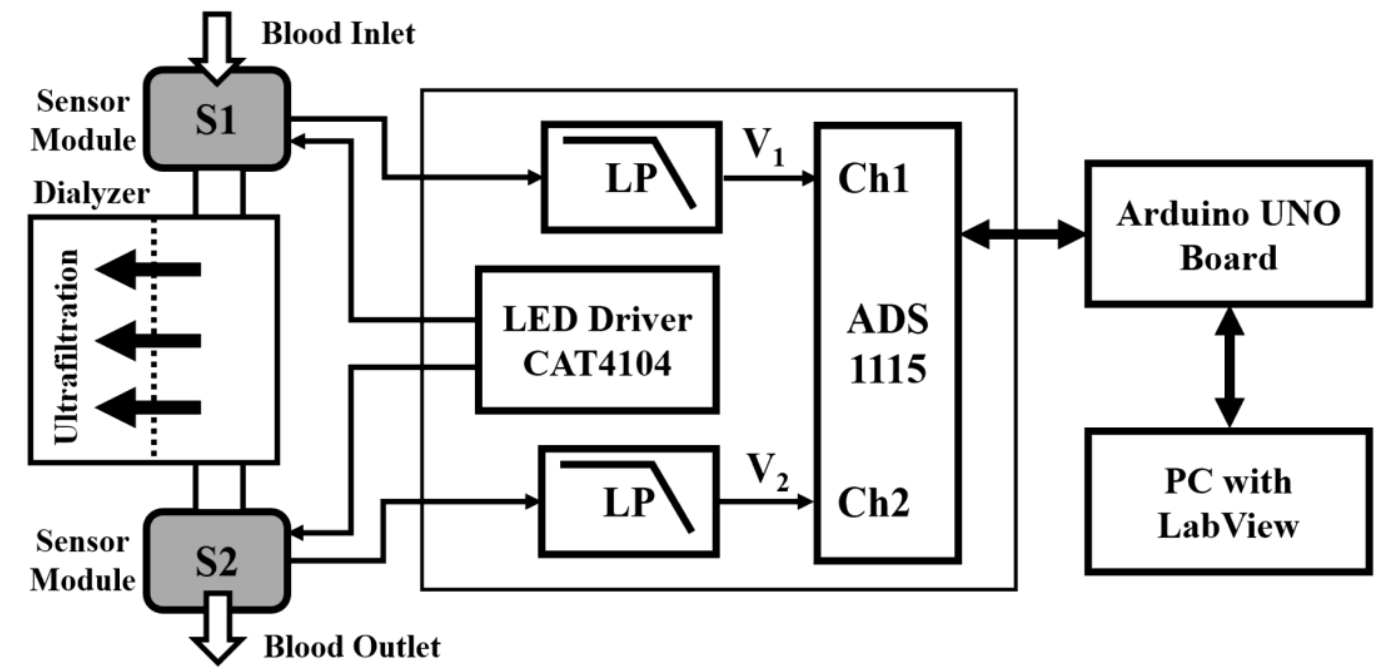

Fig. 4. Diagram of the measurement system. Two sensor modules are placed before and after the dialyzer. A PCB manages current supply to the sensor module and data sampling, together with an Arduino board. Data is sent to a PC and recorded using LabView. 


\section{Experimental protocols and data analysis}

A commercial machine for AKI treatment (Prisma, Gambro/Hospal, Medolla, Italy) was used to test the system. Its disposable line was cut to insert the optical flow-through cuvette chosen for our setup and afterwards re-sealed using cyclohexanone. The machine was set in slow continuous ultrafiltration (SCUF) mode. Blood flow rate was set to 100 $\mathrm{ml} / \mathrm{min}$. Three liters of rabbit blood were placed in a container and kept from coagulating using sodium citrate at the appropriate concentration of $4 \% \mathrm{w} / \mathrm{v}$ at $1: 9$ dilution and two experiments were performed. Hematocrit was measured before and after each protocol using a capillary centrifuge (Ematomed, Angelo Franceschini Srl, Italy).

During the first experiment, ultrafiltration rate (UFR) was changed manually using the machine's touchscreen in a progressively increasing and then decreasing manner (Fig. 5, top left panel). Steps of $50 \mathrm{ml} / \mathrm{h}$ were applied to UFR, starting from $10 \mathrm{ml} / \mathrm{h}$ up to $210 \mathrm{ml} / \mathrm{h}$ and then back to $10 \mathrm{ml} / \mathrm{h}$. Each UFR value was kept constant for 3 minutes for a total protocol duration of 27 minutes. This protocol aimed at investigating the relationship between UFR and the optical signals. The second experiment followed the general concept of the first one by increasing and decreasing UFR in steps. However, in this case each transition was interleaved with a return step to $10 \mathrm{ml} / \mathrm{h}$. UFR steps were taken by starting from $10 \mathrm{ml} / \mathrm{h}$ and going to 60, 150 and $210 \mathrm{ml} / \mathrm{h}$ and back (Fig. 5, top right panel). Steps were 3-minutes long, bringing the total duration of this experiment to 39 minutes. The aims of this protocol were to investigate the possible presence of a baseline drift in
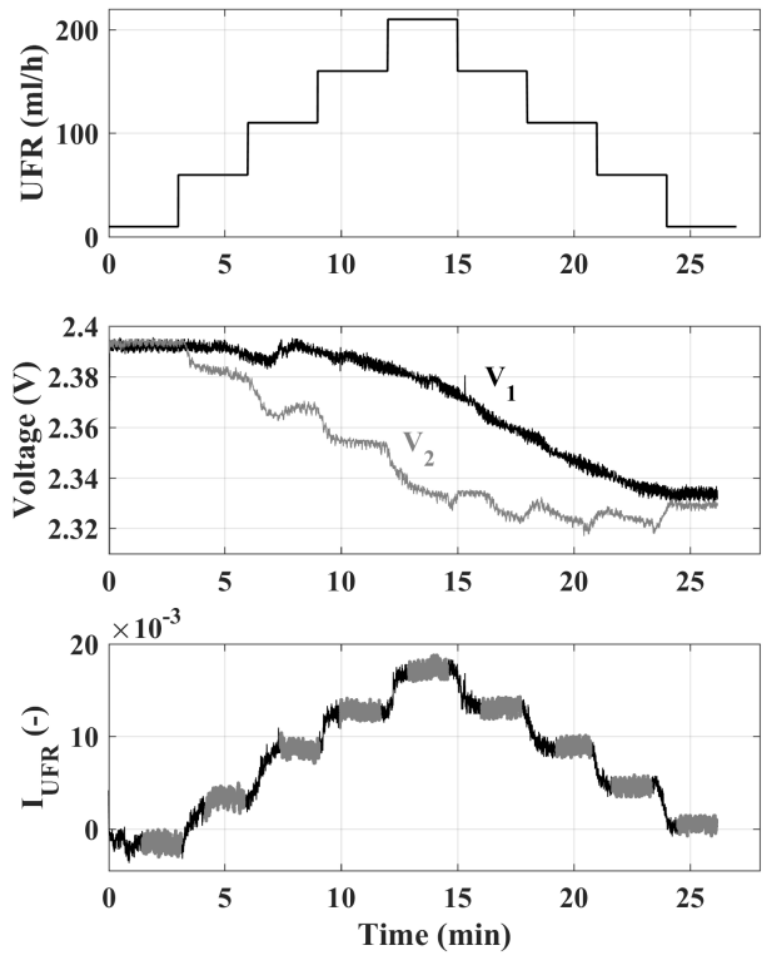

the system and to collect additional data for the estimation of the UFR/optical relationship.

Signal processing and data analysis were performed using Matlab (Mathworks, Natick, Massachussets, USA).

Signals $V_{1}$ and $V_{\text {Diff }}$ were first pre-processed using a finiteimpulse response (FIR) filter of the 20th order with a cut-off frequency of $0.6 \mathrm{~Hz}$, in order to remove the blood-mediated oscillating effect of the machine's roller pump on the optical signal. After filtering the two signals, IUFR was calculated according to Eq. (9) as $\mathrm{V}_{\text {Diff }} / \mathrm{V}_{1}$.

From the available data, time intervals were selected for analysis from each 3-minute step; while for each UFR step, a 100 -second time interval was selected.

Intervals had to be selected manually to account for:

- mismatch between the timing of the protocol and the operator-performed change in UFR

- the time delay necessary for the machine to speed up or slow down the pump responsible for ultrafiltration.

For each interval, the mean IUFR was computed. Afterwards, linear regression between the average $\mathrm{I}_{\mathrm{UFR}}$ values and the corresponding UFR was performed. From the linear regression coefficients, the estimated value of UFR was computed and compared with the reference value.

\section{RESUlTS}

In protocol \#1, hematocrit was measured before and after the experiments at $32 \%$, indicating an overall change of less than $1 \%$. In protocol \#2, hematocrit was $32 \%$ before the experiment and $33 \%$ after, due to the higher total


Fig. 5. Results from the experimental measurements. Left column panels show data for protocol \#1, right column panels show data from protocol \#2. Top panels show reference UFR values set on the machine's user interface. Middle panels show measured values for $V_{1}$ (black) and $V_{2}$ (grey). Lower panels show the computed value of $\mathrm{I}_{\mathrm{UFR}}$ as black lines, whereas the subset of data used for linear regression is shown in grey. 
ultrafiltration involved in this protocol.

Fig. 5 shows the experimental results for both protocols. The middle panels show the trend of signal $\mathrm{V}_{1}$ and $\mathrm{V}_{2}$ in response to the UFR values shown in the top panels. It is clear from these graphs that signal $\mathrm{V}_{2}$, measured post-filter, changes in response to a change in UFR. This is consistent with our model reported in Eq. (7), as UFR creates a mismatch in hemoglobin concentration across the filter that causes a difference between signals $\mathrm{V}_{1}$ and $\mathrm{V}_{2}$. A decreasing trend is also observable in both signals: in $\mathrm{V}_{1}$ it is almost linear, whereas in $V_{2}$ is it superimposed to the step responses. The brief signal spike in $\mathrm{V}_{2}$ in protocol \#2 (middle-right panel, grey line, time $28-30 \mathrm{~min}$ ) is due to an erroneous stop of the blood flow, quickly restarted.

The drift present in $\mathrm{V}_{1}$ in both protocols can be explained by the effect of recirculation: after ultrafiltration at the dialyzer, concentrated blood flows back to the container, mixes with the original pool, and re-enters the filter. Thus, the average value of $[\mathrm{Hgb}]_{1}$ will slightly increase over time decreasing $\mathrm{V}_{1}$. This effect should also be noticeable in actual patients, although the presence of other body compartments would counteract strong volume reductions with refilling.

The recorded tracks for $\mathrm{V}_{1}$ and $\mathrm{V}_{2}$ also show that, for the UFR range tested, $V_{\text {Diff }}$ is in the order of tens of milliVolts. Thus, the choice of measuring $\mathrm{V}_{\text {Diff }}$ directly with a differential amplifier configuration is justified in order to record such a small signal with improved resolution.

Lower panels of Fig. 5 show the computed value for IUFR. For both protocols, the trend of IUFR shows a remarkable similarity with the reference UFR track reported in the upper left and right panels, supporting the hypothesis that $\mathrm{I}_{\text {UFR }}$ can be used to estimate UFR from the optical data.

Moreover, whereas signals $\mathrm{V}_{1}$ and $\mathrm{V}_{2}$ showed a trend of slow decrease due to the recirculation of concentrated blood, IUFR shows negligible signs of this effect. This is confirmed by the fact that the $\mathrm{I}_{\mathrm{UFR}}$ value in response to the baseline UFR level of $10 \mathrm{ml} / \mathrm{min}$ does not drift significantly. In fact, IUFR is virtually only sensitive to the effect of UFR steps.

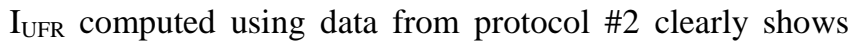
the presence of a time-dependent first-order step response. A

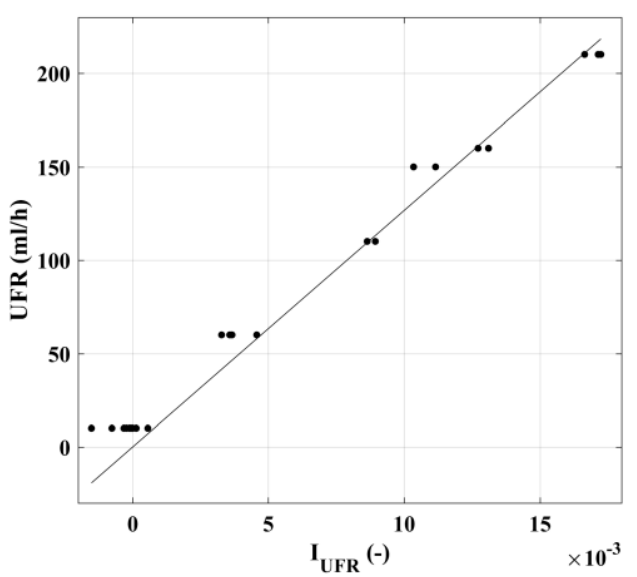

Fig. 6. Linear regression between reference UFR values and index $\mathrm{I}_{\mathrm{UFR}}$, shown by the black line. Data points from experimental measurements are represented by black dots. likely explanation is that this behaviour is more evident in protocol \#2 because the change in UFR is larger between consecutive steps and the machine's ultrafiltration mechanism requires more time to set UFR to the value requested by the operator.

In Fig. 6, results from linear regression between the reference UFR value and our index $\mathrm{I}_{\text {UFR }}$ are shown. The linear regression process returned an $R^{2}$ value of 0.97 . This high $R^{2}$ value confirms the validity of fitting the data with a linear model. Comparing the UFR values predicted by the regression model with the reference UFR values yielded a mean \pm std error of $-7.7 \pm 9.9 \mathrm{ml} / \mathrm{h}$ and an $\mathrm{rms}$ error of $12.4 \mathrm{ml} / \mathrm{h}$, equivalent to $0.21 \mathrm{ml} / \mathrm{min}$.

\section{DISCUSSION AND CONCLUSION}

\section{A. Analysis of the Results}

The first quantitative result of this work is the very high correlation found between the reference value of UFR and the IUFR index. Linear correlation between the two variables was also expected from theory, as reported in Section III.A., and these results demonstrate the sound theoretical basis of this work.

Another quantitative result is the low rms error yielded when predicting UFR by linear regression from $\mathrm{I}_{\mathrm{UFR}}$, which is $\simeq 12 \mathrm{ml} / \mathrm{h}$. This result indicates that our system has an accuracy approximately double to that of the UFR setting reported in the machine's manual $(30 \mathrm{ml} / \mathrm{h})[14]$. However, it should be mentioned that the UFR setting on the machine is also our UFR reference value for the linear regression. Thus, since the machine is also our gold standard, it cannot be formally demonstrated that our system has better accuracy, only equal. Nonetheless, the results obtained with this proof-of-concept setup show that our sensor system could be a valid replacement for the current state-of-the-art UFR monitoring method, with comparable or even better accuracy.

In addition to the highly accurate monitoring of UFR, our system shows potential for another application: by numerical integration of the estimated UFR value, the total UF volume removed could be estimated at any moment during the session. This capability would significantly simplify the machine as there would be no more need for an electronic scale, although waste outflow by a bag or tubing would still be necessary. However, this additional improvement is dependent on the task of removing the systematic part of the estimation error, in order to avoid long-term drifts in the value of the total estimated UF volume. This could be accomplished by periodic calibration of the sensors performed during short periods at zero or minimum, known UFR.

The main strengths of this system are its cheapness, ruggedness and simplicity: whereas other optical flow measurement techniques like laser doppler or particle image velocimetry could be employed, they would require more complex, expensive and/or delicate setups.

The robustness and compact size of our system also means it could be integrated into smaller blood purification systems currently being developed, such as portable artificial kidneys, 
an area attracting growing interest.

Ultrafiltration can also be used to treat some types of heart failure[11], [13]; another possible target application for this system in addition to AKI/RRTs.

\section{B. Limitations and Future Developments}

The sensor system has some limitations, which at present have negligible effect on the quality of the work but will be nonetheless addressed in future stages of the project:

1. The main limitation of this work is that additional factors that may influence the optical measurements, and thus the relationship with UFR, have not yet been investigated in these studies. Examples of such factors are the level of blood dilution and the blood flow rate, which have been kept constant in our experiment.

2. Data for correlation was chosen manually during preprocessing. This was necessary as a commercial HD machine was used and the true instantaneous value of UFR was unknown to us. As shown in Fig. 5, data was only excluded from the transients when the set UFR value was not guaranteed and all steady-state data was kept.

3. Only one experimental session, comprised of two experiments with different protocols, has been conducted. However, results show that the collected data is in accordance with the theoretical part of the work, and a strong linear correlation exists between the measured quantity, the IUFR index, and the ultrafiltration rate UFR. Overall, the quality of collected data is very high for one experimental session.

4. LED sources in the setup were powered with constant current without any modulation scheme. However, care was taken to shield sensor housings from environmental light. Moreover, no slow long-term drift in light source power may have influenced our results since data was collected using a dynamic protocol with UFR steps.

In future developments of this work, additional experimental sessions will be conducted, both to enlarge the dataset collected with the existing protocols, and to investigate the effect of the abovementioned additional factors. The new measurement circuit will also include Pulse-Width Modulation (PWM) for the light sources. If available, a setup with UFR value known in real time will be used.

\section{Conclusion}

In conclusion, a simple yet highly performing non-invasive sensor system has been developed to estimate ultrafiltration rate in ultrafiltration-based blood purification therapies such as HF and SCUF. The system is simple, rugged, low-cost and operates on sound theoretical foundations. Compared to stateof-the-art systems, it has improved accuracy and the potential to drastically simplify the architecture needed in blood purification machines to perform UF control.

\section{REFERENCES}

[1] J. A. Sargent and Gotch F.A., "Principles and biophysics of dialysis," in Replacement of Renal Function by Dialysis, Springer., W. Drukker, F.M. Parsons, J.F. Maher, 1983.

[2] C. Ronco, P. M. Ghezzi, A. Brendolan, C. Crepaldi, and G. La Greca, "The haemodialysis system: basic mechanisms of water and solute transport in extracorporeal renal replacement therapies," Nephrol. Dial. Transplant. Off. Publ. Eur. Dial. Transpl. Assoc. Eur. Ren. Assoc., vol. 13 Suppl 6, pp. 3-9, 1998.

[3] A. Nissenson and R. N. Fine, Clinical Dialysis, Fourth Edition. McGraw Hill Professional, 2005.

[4] J. Waniewski, "Peritoneal fluid transport: mechanisms, pathways, methods of assessment," Arch. Med. Res., vol. 44, no. 8, pp. 576-583, Nov. 2013.

[5] D. Journois, Continuous Hemofiltration in the Intensive Care Unit. CRC Press, 1997.

[6] C. Ronco, R. Bellomo, and J. A. Kellum, Critical Care Nephrology. Elsevier Health Sciences, 2009.

[7] E. P. Paganini, Acute Continuous Renal Replacement Therapy. Springer Science \& Business Media, 2012.

[8] M. Ursino et al., "A mathematical model for the prediction of solute kinetics, osmolarity and fluid volume changes during hemodiafiltration with on-line regeneration of ultrafiltrate (HFR)," Int. J. Artif. Organs, vol. 29, no. 11, pp. 1031-1041, Nov. 2006.

[9] G. Casagrande et al., "Patient-specific modeling of multicompartmental fluid and mass exchange during dialysis," Int. J. Artif. Organs, vol. 39, no. 5, pp. 220227, Jul. 2016.

[10] E. Ravagli, E. Grandi, P. Rovatti, and S. Severi, "Finiteelement modeling of time-dependent sodium exchange across the hollow fiber of a hemodialyzer by coupling with a blood pool model," Int. J. Artif. Organs, vol. 39, no. 9, pp. 471-478, Nov. 2016.

[11] C. Ronco, Z. Ricci, R. Bellomo, and F. Bedogni, "Extracorporeal Ultrafiltration for the Treatment of Overhydration and Congestive Heart Failure," Cardiology, vol. 96, no. 3-4, pp. 155-168, 2001.

[12] F. Nalesso, F. Garzotto, and C. Ronco, "Technical Aspects of Extracorporeal Ultrafiltration: Mechanisms, Monitoring and Dedicated Technology," Fluid Overload, vol. 164, pp. 199-208, 2010.

[13] M. R. Costanzo et al., "Extracorporeal Ultrafiltration for Fluid Overload in Heart Failure," J. Am. Coll. Cardiol., vol. 69, no. 19, pp. 2428-2445, May 2017.

[14] R. Bellomo and C. Ronco, "Continuous renal replacement therapy in the intensive care unit," Intensive Care Med., vol. 25, no. 8, pp. 781-789, Aug. 1999.

[15] “Prisma System Operator's Manual." - Available at http://www.crrtonline.com/docs/OperatorsPrismaEng.pd f - Last accessed July 7, 2018.

[16] S. Cattini and L. Rovati, "An Optical Technique for Real-Time Monitoring of Hemolysis During Hemodialysis," IEEE Trans. Instrum. Meas., vol. 65, no. 5, pp. 1060-1069, May 2016. 
[17] M. K. Sharma, F. P. Wieringa, A. J. H. Frijns, and J. P. Kooman, "On-line monitoring of electrolytes in hemodialysis: on the road towards individualizing treatment," Expert Rev. Med. Devices, vol. 13, no. 10, pp. 933-943, Oct. 2016.

[18] E. Ravagli, M. Crescentini, M. Tartagni, and S. Severi, "Non-invasive measurement of electrical conductivity of liquids in biocompatible polymeric lines for hemodialysis applications," Sens. Actuators Phys., vol. 261, no. Supplement C, pp. 252-260, Jul. 2017.

[19] M. K. Sharma et al., "A Fluorescent Micro-Optofluidic Sensor for In-Line Ion Selective Electrolyte Monitoring," IEEE Sens. J., vol. 18, no. 10, pp. 39463951, May 2018.

[20] F. Uhlin, I. Fridolin, L.-G. Lindberg, and M. Magnusson, "Estimation of delivered dialysis dose by on-line monitoring of the ultraviolet absorbance in the spent dialysate," Am. J. Kidney Dis. Off. J. Natl. Kidney Found., vol. 41, no. 5, pp. 1026-1036, May 2003.

[21] E. Ravagli and S. Severi, "Optical and Electrical Characterization of Biocompatible Polymeric Lines for Hemodialysis Applications," Materials, vol. 11, no. 3, p. 438, Mar. 2018.

[22] F. Paolini, E. Mancini, A. Bosetto, and A. Santoro, "Hemoscan: a dialysis machine-integrated blood volume monitor," Int. J. Artif. Organs, vol. 18, no. 9, pp. 487-494, Sep. 1995.

[23] J. J. Dasselaar, R. M. Huisman, P. E. DE Jong, and C. F. M. Franssen, "Relative blood volume measurements during hemodialysis: comparisons between three noninvasive devices," Hemodial. Int. Int. Symp. Home Hemodial., vol. 11, no. 4, pp. 448-455, Oct. 2007.

[24] S. Sbrignadello, G. Pacini, and A. Tura, "Determination of Glucose Levels during Dialysis Treatment: Different Sensors and Technologies," Journal of Sensors, 2016. [Online].

[25] E. Mancini et al., "Intra-dialytic blood oxygen saturation (SO2): association with dialysis hypotension (the SOGLIA Study)," J. Nephrol., vol. 30, no. 6, pp. 811-819, Dec. 2017.

[26] D. Schneditz et al., "Nature and rate of vascular refilling during hemodialysis and ultrafiltration," Kidney Int., vol. 42, no. 6, pp. 1425-1433, Dec. 1992.

[27] S. Cavalcanti et al., "Model-based study of the effects of the hemodialysis technique on the compensatory response to hypovolemia," Kidney Int., vol. 65, no. 4, pp. 1499-1510, Apr. 2004.

[28] S. Severi et al., "Cardiac response to hemodialysis with different cardiovascular tolerance: heart rate variability and QT interval analysis," Hemodial. Int. Int. Symp. Home Hemodial., vol. 10, no. 3, pp. 287-293, Jul. 2006.

[29] J. J. Dasselaar, "Relative blood volume based biofeedback during haemodialysis," J. Ren. Care, vol. 33, no. 2, pp. 59-65, Jun. 2007.

[30] F. Javed, A. V. Savkin, G. S. H. Chan, J. D. Mackie, and N. H. Lovell, "Identification and Control for Automated Regulation of Hemodynamic Variables During Hemodialysis," IEEE Trans. Biomed. Eng., vol. 58, no. 6, pp. 1686-1697, Jun. 2011.
[31] A. Santoro, E. Mancini, and A. T. Azar, "Biofeedback Systems and Their Application in the Hemodialysis Therapy," in Modeling and Control of Dialysis Systems, Springer, Berlin, Heidelberg, 2013, pp. 1081-1107.

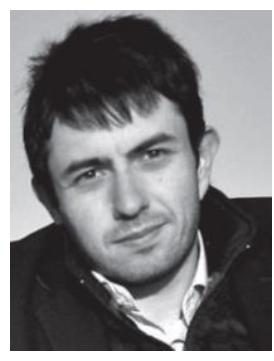

Andrea Visotti - B.Sc in Biomedical Engineering 2009, M.Sc. 2012, University of Bologna, Italy. R\&D Coordinator at CA-MI Srl, 2012-2014, where he filed several patents and published a scientific article. Cofounded IBD Srl in 2014, where he is currently R\&D Coordinator.

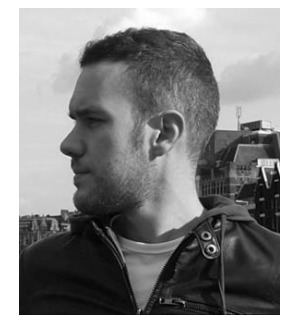

Enrico Ravagli - PhD in Bioengineering, 2017, University of Bologna, Italy, where he is currently a postdoctoral researcher. His research activities are focused on non-invasive sensing and estimation methods for hemodialysis and extracorporeal circulation applications.



Claudia Perazzini - B.Sc. in Biomedical Engineering 2007, M.Sc. 2010, University of Bologna, Italy, where between 2011 and 2016 she worked as a research fellow at the Health Sciences and Technologies Interdepartmental Centre for Industrial Research. Currently in R\&D at IBD srl.

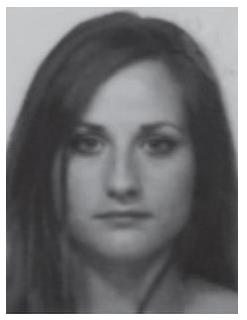

Debora Drudi - M.Sc Biomedical Engineering, 2016, University of Bologna. Currently employed at IBD Srl as Regulatory \& Quality Manager, monitoring compliance of company processes and standards. Responsible for activities concerning the attainment of the CE mark.

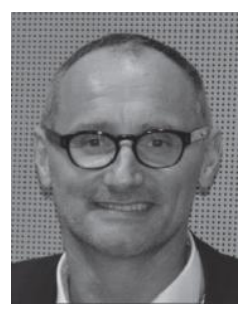

Corrado Ghidini - Doctor in Dental Science (DDS), Doctor of Medicine (M.D.), Specialization in Internal Medicine, 19811993, University of Bologna. Founder and CEO of two of the highest ranked dental practices in Italy. Founder, $\mathrm{CEO}$ and $\mathrm{CFO}$ of IBD Srl.

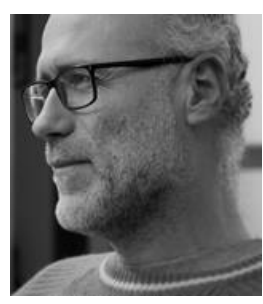

Stefano Severi - M.Sc. in Electronic Engineering and $\mathrm{PhD}$ in Bioengineering, University of Bologna, Italy. Assistant Professor at the Department of Electrical, Electronic and Information Engineering, University of Bologna, 1998-present. Head of the Computational Physiopathology Unit, with research focusing on computational cardiology, artificial kidney and hemodialysis therapy. 